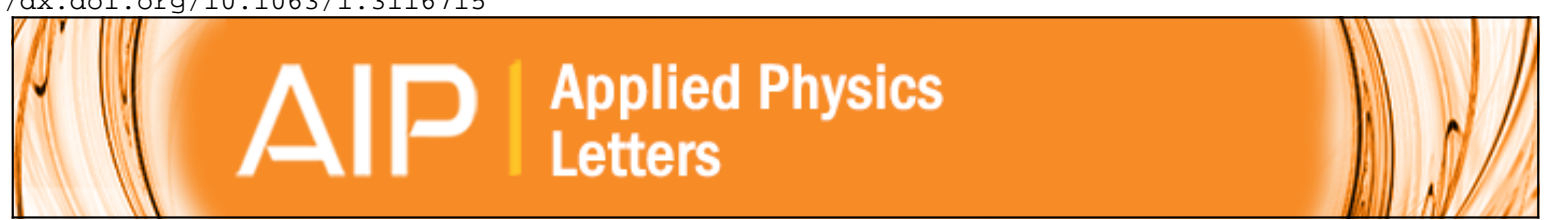

\title{
Thermal transport in composites of self-assembled nickel nanoparticles embedded in yttria stabilized zirconia
}

Nitin C. Shukla, Hao-Hsiang Liao, Jeremiah T. Abiade, Mitsuhiro Murayama, Dhananjay Kumar, and Scott T. Huxtable

Citation: Applied Physics Letters 94, 151913 (2009); doi: 10.1063/1.3116715

View online: http://dx.doi.org/10.1063/1.3116715

View Table of Contents: http://scitation.aip.org/content/aip/journal/apl/94/15?ver=pdfcov

Published by the AIP Publishing

\section{Articles you may be interested in}

Orientation dependence of thermal conductivity in copper-graphene composites

J. Appl. Phys. 110, 074901 (2011); 10.1063/1.3641640

The grain size and temperature dependence of the thermal conductivity of polycrystalline, tetragonal yttriastabilized zirconia

Appl. Phys. Lett. 98, 211906 (2011); 10.1063/1.3593383

Laser ablation characteristics of yttria-doped zirconia in the nanosecond and femtosecond regimes

J. Appl. Phys. 107, 014908 (2010); 10.1063/1.3275868

Thermal properties of the optically transparent pore-free nanostructured yttria-stabilized zirconia

J. Appl. Phys. 106, 113507 (2009); 10.1063/1.3264613

Oxygen sensing junctions based on yttria stabilized zirconia with platinum nanoparticles

Rev. Sci. Instrum. 77, 056108 (2006); 10.1063/1.2202925

\section{AAP Re-register for Table of Content Alerts}




\title{
Thermal transport in composites of self-assembled nickel nanoparticles embedded in yttria stabilized zirconia
}

\author{
Nitin C. Shukla, ${ }^{1}$ Hao-Hsiang Liao, ${ }^{1}$ Jeremiah T. Abiade, ${ }^{1,2}$ Mitsuhiro Murayama, ${ }^{3}$ \\ Dhananjay Kumar, ${ }^{4,5}$ and Scott T. Huxtable ${ }^{1, a)}$ \\ ${ }^{1}$ Department of Mechanical Engineering, Virginia Polytechnic Institute and State University, Blacksburg, \\ Virginia 24061, USA \\ ${ }^{2}$ Department of Materials Science and Engineering, Virginia Polytechnic Institute and State University, \\ Blacksburg, Virginia 24061, USA \\ ${ }^{3}$ Institute for Critical Technology and Applied Science, Virginia Polytechnic Institute and State University, \\ Blacksburg, Virginia 24061, USA \\ ${ }^{4}$ Department of Mechanical and Chemical Engineering and Center for Advanced Materials and Smart \\ Structures (CAMSS), North Carolina A\&T State University, Greensboro, North Carolina 27411, USA \\ ${ }^{5}$ Condensed Matter Sciences Division, Oak Ridge National Laboratory (ORNL), Oak Ridge, \\ Tennessee 37831, USA
}

(Received 24 December 2008; accepted 16 March 2009; published online 17 April 2009)

\begin{abstract}
We investigate the effect of nickel nanoparticle size on thermal transport in multilayer nanocomposites consisting of alternating layers of nickel nanoparticles and yttria stabilized zirconia (YSZ) spacer layers that are grown with pulsed laser deposition. Using time-domain thermoreflectance, we measure thermal conductivities of $k=1.8,2.4,2.3$, and $3.0 \mathrm{~W} \mathrm{~m}^{-1} \mathrm{~K}^{-1}$ for nanocomposites with nickel nanoparticle diameters of 7,21,24, and $38 \mathrm{~nm}$, respectively, and $k=2.5 \mathrm{~W} \mathrm{~m}^{-1} \mathrm{~K}^{-1}$ for a single $80 \mathrm{~nm}$ thick layer of YSZ. We use an effective medium theory to estimate the lower limits for interface thermal conductance $G$ between the nickel nanoparticles and the YSZ matrix $\left(G>170 \mathrm{MW} \mathrm{m}^{-2} \mathrm{~K}^{-1}\right)$, and nickel nanoparticle thermal conductivity. (C) 2009 American Institute of Physics. [DOI: 10.1063/1.3116715]
\end{abstract}

Materials composed of self-assembled nanoparticles embedded in a matrix material exhibit interesting physical properties. The inclusion of nanoparticles in composite materials may result in unique or improved mechanical, optical, electrical, thermal, and magnetic properties. ${ }^{1-3}$ These composite materials may find potential applications in data storage devices, biochemical sensors, magnetic field sensors, and single electron devices. ${ }^{4,5}$ In this work, we study the effect of nanoparticle size on thermal transport in a series of nanocomposites that consist of layers of nickel nanoparticles embedded in a yttria stabilized zirconia (YSZ) matrix. A better understanding of heat transport in nanostructured composite materials is important not only from a scientific viewpoint but also to improve the performance of many of the abovementioned devices.

Nickel nanoparticles are embedded in polycrystalline YSZ films by laser ablation of Ni and YSZ (5 wt \% yttria) targets using pulsed laser deposition. ${ }^{6}$ To avoid the formation of $\mathrm{NiO}$ or $\mathrm{Ni} / \mathrm{NiO}$ core/shell structures, oxygen is not used and the depositions are done in vacuum $\left(\sim 5 \times 10^{-7}\right.$ Torr $)$. The final structure of the multilayered nanocomposites consists of an initial YSZ buffer layer $(\sim 100 \mathrm{~nm})$ followed by five Ni nanoparticle layers $(7-38 \mathrm{~nm})$ that are separated by four YSZ spacer layers $(22-48 \mathrm{~nm})$, as shown in Fig. 1. The $\mathrm{Ni}$ particle size is altered by varying the number of laser pulses at a laser frequency of $10 \mathrm{~Hz}$.

Microstructural investigations are carried out using a FEI TITAN 80-300 equipped with an E.A. Fischione model 3000 annular dark field detector and operated at $200 \mathrm{kV}$ in scanning transmission electron microscopy (STEM) mode. The size of the electron probe is approximately $1 \mathrm{~nm}$ in

${ }^{a)}$ Electronic mail: huxtable@vt.edu. diameter. From STEM dark field images of these four samples (see Fig. 1) we determine the average Ni nanoparticle diameters to be approximately 7, 21, 24, and $38 \mathrm{~nm}$ for samples 1-4, respectively. While these particular samples were not analyzed with electron energy loss spectroscopy (EELS), similar samples grown by the same group were examined with EELS and pure metallic Ni nanoparticles without oxide layers were found. ${ }^{7}$

We use time-domain thermoreflectance (TDTR) (Ref. 8) to measure the thermal conductivity $k$ and interface thermal conductance $G$ of the nanocomposites. TDTR is a pump-

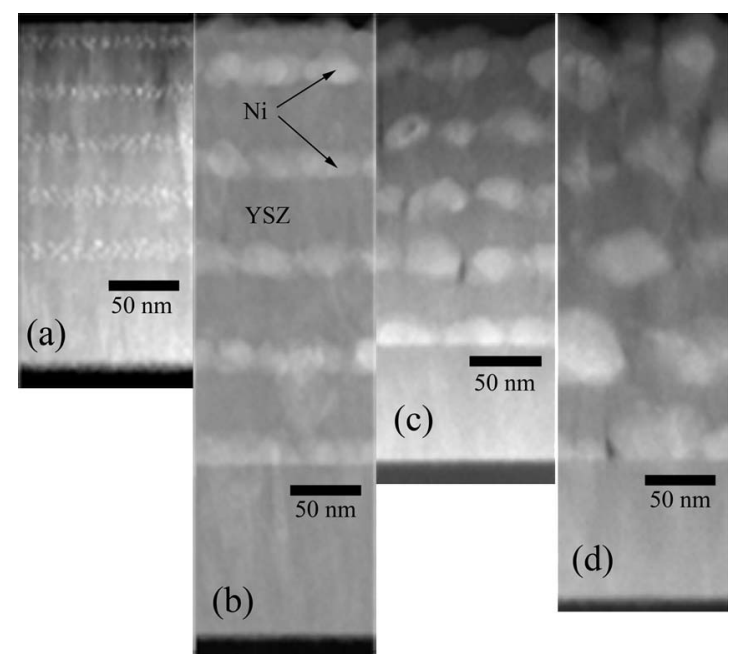

FIG. 1. Scanning transmission electron micrographs of the Ni/YSZ nanocomposites. Each sample consists of five layers of Ni nanoparticles separated by four YSZ spacer layers and they are grown on Si substrates with YSZ buffer and cap layers. The dimensions of the samples are given in Table I. Images (a)-(d) correspond to samples 1-4, respectively. 
TABLE I. Summary of properties for each sample. The effective thermal conductivity of the Ni/YSZ composite layers is extracted with TDTR. The overall thermal conductivity of the nanocomposite is calculated using a series thermal model in conjunction with our measurements on YSZ and the Ni/YSZ composite layers.

\begin{tabular}{ccccc}
\hline \hline Sample & $\begin{array}{c}\text { Diameter of Ni nanoparticles } \\
(\mathrm{nm})\end{array}$ & $\begin{array}{c}\text { Thickness of YSZ spacer } \\
(\mathrm{nm})\end{array}$ & $\begin{array}{c}\text { Effective } k \text { of Ni/YSZ composite layer } \\
\left(\mathrm{W} \mathrm{m}^{-1} \mathrm{~K}^{-1}\right)\end{array}$ & $\begin{array}{c}\text { Overall } k \text { of nanocomposite structure } \\
\left(\mathrm{W} \mathrm{m}^{-1} \mathrm{~K}^{-1}\right)\end{array}$ \\
\hline 1 & 7 & 22 & 1.3 & 1.8 \\
2 & 21 & 48 & 2.2 & 2.4 \\
3 & 24 & 22 & 2.1 & 2.3 \\
4 & 38 & 28 & 3.5 & 3.0 \\
\hline \hline
\end{tabular}

probe optical technique that takes advantage of the fact that the reflectivity of a metal has a slight dependence on temperature. A thin film of aluminum $(\sim 100 \mathrm{~nm})$ with high thermoreflectance at $800 \mathrm{~nm}$ is deposited on top of all four nanocomposite samples using electron beam evaporation. Ultrashort Ti:sapphire laser pulses with repetition rate of 80 $\mathrm{MHz}$ and wavelength of $800 \mathrm{~nm}$ are split into pump and probe beams using a 50:50 beamsplitter. The pump beam heats the sample and is modulated at $9.8 \mathrm{MHz}$, while the time-delayed probe beam gives a measure of the reflectivity changes, and therefore temperature decay, at the surface of the sample. The $1 / e^{2}$ beam diameters at the sample are $30 \mu \mathrm{m}$ for the pump beam and $18 \mu \mathrm{m}$ for the probe beam. Data are recorded with a lock-in amplifier in order to improve the signal-to-noise ratio.

The ratio of the in-phase voltage to the out-of-phase voltage measured by the lock-in amplifier is compared with a one dimensional heat transfer model for multilayer structures to extract thermal conductivity and interface thermal conductance. ${ }^{9}$ Since the TDTR model is valid only for layers of uniform thickness, we represent the Ni nanoparticle layer as a Ni/YSZ composite layer with the layer thickness set equal to the diameter of the Ni nanoparticles. This Ni/YSZ composite layer is approximated as a layer of uniformly arranged spherical Ni nanoparticles surrounded by YSZ. Thus the overall structure in the model consists of alternating $\mathrm{Ni}$ / YSZ composite layers separated by YSZ spacer layers. For spherical nanoparticles, the maximum volume fraction within the Ni/YSZ composite layer is given by the case where each nanoparticle is touching four neighboring $\mathrm{Ni}$ nanoparticles and the volume fraction $f$ of Ni in YSZ is then $f=\pi / 6$, or $\sim 52.4 \%$.

The TDTR model requires the thickness, heat capacity, and thermal conductivity of each layer, along with the interface thermal conductance between the layers in our nanocomposite structure, as input parameters. The thickness of the aluminum layer is calculated using measurements of the acoustic echoes ${ }^{10}$ with the speed of sound for Al taken as $6420 \mathrm{~m} / \mathrm{s}$ (Ref. 11). The YSZ and Ni/YSZ composite layer thicknesses are measured using the TEM. The heat capacities of $\mathrm{Al}, \mathrm{Ni}$, and $\mathrm{Si}$ are taken from literature values, ${ }^{11,12}$ while the heat capacity of YSZ is calculated using literature values for the constituent materials $\mathrm{Y}_{2} \mathrm{O}_{3}$ (Ref. 13) and $\mathrm{ZrO}_{2}$ (Ref. 14). The heat capacity of the Ni/YSZ composite layer is then calculated using the Kopp-Neumann law assuming the volume fraction of $\mathrm{Ni}$ to be $\pi / 6$. We point out that the uncertainty in the heat capacity of the Ni/YSZ composite layer resulting from uncertainty in the Ni volume fraction does not strongly influence the TDTR measurement results. For example, a $25 \%$ change in the Ni volume fraction in the TDTR model results in a change of less than $8 \%$ in the measured thermal conductivity of the Ni/YSZ composite layer. The thermal conductivity of silicon is taken from Shanks et al., ${ }^{12}$ and the thermal conductivity of the aluminum layer is found to be $185 \mathrm{~W} \mathrm{~m}^{-1} \mathrm{~K}^{-1}$ using four-point probe electrical conductivity measurements in conjunction with the WiedemannFranz law.

Thus, the only unknown parameters in our TDTR model are: $G$ between $\mathrm{Al}$ and YSZ, $k$ of YSZ, and the effective $k$ of the Ni/YSZ composite layer. This effective $k$ includes the interface thermal conductance between the Ni nanoparticles and the YSZ matrix, which is discussed later. In order to reduce the number of unknown parameters, we first measure $k$ for YSZ and $G$ between $\mathrm{Al}$ and YSZ by preparing a reference sample that consists of $80 \mathrm{~nm}$ of YSZ deposited on a $\mathrm{Si}$ substrate. Using TDTR, we obtain $k=2.5 \mathrm{~W} \mathrm{~m}^{-1} \mathrm{~K}^{-1}$ for YSZ and $G=100 \mathrm{MW} \mathrm{m}^{-2} \mathrm{~K}^{-1}$ for the $\mathrm{Al} / \mathrm{YSZ}$ interface. This experimental value of $k$ for YSZ is similar to values of $2.3-3.0 \mathrm{~W} \mathrm{~m}^{-1} \mathrm{~K}^{-1}$ reported in the literature. ${ }^{15,16}$

Next, we use the above values to extract the effective $k$ for the Ni/YSZ composite layers and we find $k$ to be $1.3,2.2$, 2.1, and $3.5 \mathrm{~W} \mathrm{~m}^{-1} \mathrm{~K}^{-1}$ in samples $1-4$, respectively. In order to determine the overall thermal conductivity of the entire nine layer structure, we create a simple series thermal resistance model that consists of the five Ni/YSZ composite layers in series with the four YSZ spacer layers. When we divide the total thickness of the nine layers by the total thermal resistance of the same layers, we find the overall thermal conductivities of the entire multilayer structures to be 1.8 , $2.4,2.3$, and $3.0 \mathrm{~W} \mathrm{~m}^{-1} \mathrm{~K}^{-1}$ for samples $1-4$, respectively (see Table I). Since the thermal conductivity of bulk nickel is $\sim 90 \mathrm{~W} \mathrm{~m}^{-1} \mathrm{~K}^{-1}$ (Ref. 11), thermal models that ignore interface effects would predict an increase in the thermal conductivity with the addition of a higher thermal conductivity material in a low thermal conductivity matrix. However, we observe a decrease in the thermal conductivity for samples 1 , 2 , and 3 that contain smaller nanoparticles. This decrease in thermal conductivity indicates that the interface thermal resistance between YSZ and $\mathrm{Ni}$ becomes dominant at these lengthscales and/or the thermal conductivity of the nickel becomes comparatively low for these nanoparticles. We address these two possibilities below.

With the use of an effective medium theory, ${ }^{17-19}$ in principle one could estimate $G$ or $k$ for the nanoparticles within these types of composite materials. In this particular instance, uncertainty in the $\mathrm{Ni}$ volume fraction makes it difficult to extract specific values for either $G$ or $k$ for the nanoparticles. However, with an estimate of the $\mathrm{Ni}$ volume fraction we can place approximate lower bounds on both properties through the use of an effective medium theory.

Every et al. ${ }^{19}$ developed the following equation for composites made from spherical particles embedded in a host 


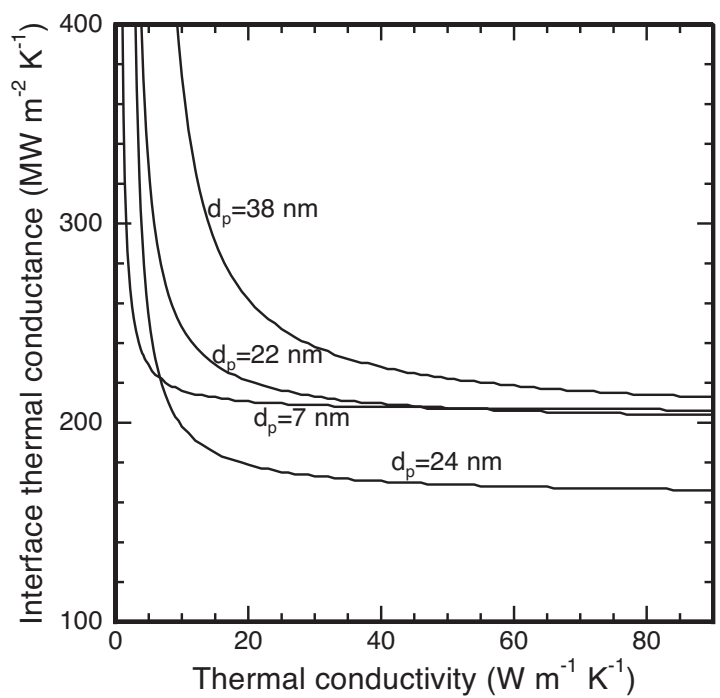

FIG. 2. Interface thermal conductance between the Ni nanoparticles and the surrounding YSZ matrix as a function of Ni nanoparticle thermal conductivity. The solid lines are predictions from an effective medium theory for each sample with a Ni volume fraction of $f=\pi / 6$. The labels on the plot refer to the average diameter of the nickel nanoparticles in each sample. We find a lower limit for the Ni/YSZ interface thermal conductance of $\sim 170 \mathrm{MW} \mathrm{m}^{-2} \mathrm{~K}^{-1}$

matrix that relates the thermal conductivity of the composite $k_{c}$ to the thermal conductivity of the host matrix $k_{m}$, the thermal conductivity of a spherical particle $k_{p}$, the interface thermal conductance $G$ between the particle and the matrix, the radius of the particle $r_{p}$, and the volume fraction $f$ of the particles for the high volume fraction limit:

$$
\begin{gathered}
(1-f)^{3}=\left(\frac{k_{m}}{k_{c}}\right)^{(1+2 \alpha) /(1-\alpha)} \times\left\{\frac{k_{c}-k_{p}(1-\alpha)}{k_{m}-k_{p}(1-\alpha)}\right\}^{3 /(1-\alpha)}, \\
\text { where } \alpha=\frac{k_{m}}{r_{p} G} .
\end{gathered}
$$

For $f=\pi / 6$, we plot the Ni/YSZ interface thermal conductance as a function of the thermal conductivity of the $\mathrm{Ni}$ nanoparticles $k_{p}$ for all four samples in Fig. 2. This plot demonstrates two interesting features. First, we find that the $\mathrm{Ni} / Y S Z$ interface thermal conductance decreases with increasing $k_{p}$, but approaches a minimum value for $k_{p}$ $>20 \mathrm{~W} \mathrm{~m}^{-1} \mathrm{~K}^{-1}$ for all four samples. In other words, the thermal resistance at the Ni/YSZ interface dominates if $k_{p}$ $>20 \mathrm{~W} \mathrm{~m}^{-1} \mathrm{~K}^{-1}$. We point out that recent molecular dynamics simulations ${ }^{20}$ predict that the thermal conductivity of nickel nanoparticles will always be above $30 \mathrm{~W} \mathrm{~m}^{-1} \mathrm{~K}^{-1}$ for nanoparticles greater than $7 \mathrm{~nm}$ in diameter. These results illustrate the critical importance of the interface if one intends to enhance the thermal conductivity of a composite material by introducing high conductivity particles. The effective medium theory also allows us to place an approximate lower bound on $G$ of $170-210 \mathrm{MW} \mathrm{m}^{-2} \mathrm{~K}^{-1}$ for the four samples, which falls in the middle of the typical range of $80<G<300 \mathrm{MW} \mathrm{m}^{-2} \mathrm{~K}^{-1}$ (Ref. 21) for many phononmediated interfaces. Gundrum et al. $^{21}$ measured $G$ to be $\sim 250 \mathrm{MW} \mathrm{m}^{-2} \mathrm{~K}^{-1}$ for a similar metal-nonmetal interface between $\mathrm{Cu}$ and $\mathrm{Al}_{2} \mathrm{O}_{3}$.

Alternatively, if we assign an upper bound on $G$ we can estimate a lower limit for the Ni nanoparticle thermal conductivity. From the literature, ${ }^{21}$ the majority of metal/ dielectric interfaces have $G<300 \mathrm{MW} \mathrm{m}^{-2} \mathrm{~K}^{-1}$ (with one notable exception ${ }^{22}$ of $G \sim 700 \mathrm{MW} \mathrm{m}{ }^{-2} \mathrm{~K}^{-1}$ for TiN/MgO). If we take $G=300 \mathrm{MW} \mathrm{m}^{-2} \mathrm{~K}^{-1}$, we find the minimum values of $k_{p}$ to be $1.6,5.8,3.9$, and $15.8 \mathrm{~W} \mathrm{~m}^{-1} \mathrm{~K}^{-1}$ for $\mathrm{Ni}$ nanoparticles with diameters of 7, 21, 24, and $38 \mathrm{~nm}$, respectively (see Fig. 2). Given that these minimum thermal conductivity values are much smaller than estimates from molecular dynamics simulations ${ }^{20}$ it seems likely that the actual $\mathrm{Ni} / \mathrm{YSZ}$ interface conductance is closer to the lower limit of $\sim 200 \mathrm{MW} \mathrm{m}^{-2} \mathrm{~K}^{-1}$.

In summary, we examined the thermal transport in nanocomposites composed of $\mathrm{Ni}$ nanoparticles embedded in a YSZ matrix. The Ni nanoparticle size and interface thermal conductance strongly influence the overall thermal conductivity of these nanocomposites. For small nanoparticles with diameters between 7 and $24 \mathrm{~nm}$, the inclusion of the nanoparticles decreases the thermal conductivity of the composite, while $38 \mathrm{~nm}$ diameter particles increase the thermal conductivity of the composite. An effective medium theory is used to place an approximate lower bound of $170 \mathrm{MW} \mathrm{m}^{-2} \mathrm{~K}^{-1}$ on the Ni/YSZ interface thermal conductance.

This work was partially supported by the United States National Science Foundation (U.S. NSF) under Grant Nos. CBET-0547122, NSF-NIRT DMR-0403480, and NSFBRIGE EEC-0824340, and by the Thomas F. and Kate Miller Jeffress Memorial Trust under Grant No. J-799. Some of this work was carried out using instruments in the Nanoscale Characterization and Fabrication Laboratory, a Virginia Tech facility operated by the Institute for Critical Technology and Applied Science, and we thank W.T. Reynolds, Jr. and J. McIntosh for their assistance with structural characterizations.

${ }^{1}$ T. Trindade, P. O'Brien, and N. L. Pickett, Chem. Mater. 13, 3843 (2001).

${ }^{2}$ P. T. Hammond, Adv. Mater. (Weinheim, Ger.) 16, 1271 (2004).

${ }^{3}$ A. P. Alivisatos, Science 271, 933 (1996).

${ }^{4}$ A. C. Balazs, T. Emrick, and T. P. Russell, Science 314, 1107 (2006).

${ }^{5}$ G. Decher, Science 277, 1232 (1997).

${ }^{6}$ J. T. Abiade, G. X. Miao, A. Gupta, A. A. Gapud, and D. Kumar, Thin Solid Films 516, 2082 (2008).

${ }^{7}$ J. T. Abiade, S. H. Oh, D. Kumar, M. Varela, S. Pennycook, H. Guo, A. Gupta, and J. Sankar, J. Appl. Phys. 104, 073910 (2008).

${ }^{8}$ D. G. Cahill, W. K. Ford, K. E. Goodson, G. D. Mahan, A. Majumdar, H. J. Maris, R. Merlin, and S. R. Phillpot, J. Appl. Phys. 93, 793 (2003).

${ }^{9}$ D. G. Cahill, Rev. Sci. Instrum. 75, 5119 (2004).

${ }^{10}$ H. T. Grahn, H. J. Maris, and J. Tauc, IEEE J. Quantum Electron. 25, 2562 (1989).

${ }^{11}$ D. R. Lide, CRC Handbook of Chemistry and Physics, 82nd ed. (CRC, Boca Raton, Florida, 2002).

${ }^{12}$ H. R. Shanks, P. D. Maycock, P. H. Sidles, and G. C. Danielson, Phys. Rev. 130, 1743 (1963).

${ }^{13}$ V. Swamy, H. J. Seifert, and F. Aldinger, J. Alloys Compd. 269, 201 (1998).

${ }^{14}$ T. Tojo, T. Atake, T. Mori, and H. Yamamura, J. Therm. Anal. Calorim. 57, 447 (1999).

${ }^{15}$ K. W. Schlichting, N. P. Padture, and P. G. Klemens, J. Mater. Sci. 36, 3003 (2001)

${ }^{16}$ D. P. H. Hasselman, L. F. Johnson, L. D. Bentsen, R. Syed, H. L. Lee, and M. V. Swain, Am. Ceram. Soc. Bull. 66, 799 (1987).

${ }^{17}$ D. P. H. Hasselman and L. F. Johnson, J. Compos. Mater. 21, 508 (1987)

${ }^{18}$ D. A. G. Bruggeman, Ann. Phys. 416, 636 (1935).

${ }^{19}$ A. G. Every, Y. Tzou, D. P. H. Hasselman, and R. Raj, Acta Metall. Mater. 40, 123 (1992).

${ }^{20}$ S. P. Yuan and P. X. Jiang, Int. J. Thermophys. 27, 581 (2006).

${ }^{21}$ B. C. Gundrum, D. G. Cahill, and R. S. Averback, Phys. Rev. B 72 245426 (2005).

${ }^{22}$ R. M. Costescu, M. A. Wall, and D. G. Cahill, Phys. Rev. B 67, 054302 (2003). 